\title{
Dynamic ATC Computation for Real-Time Power Markets
}

\author{
Ch. Venkaiah ${ }^{\dagger}$, D. M. Vinod Kumar* and K. Murali**
}

\begin{abstract}
In this paper, a novel dynamic available transfer capability (DATC) has been computed for real time applications using three different intelligent techniques viz. i) back propagation algorithm (BPA), ii) radial basis function (RBF), and iii) adaptive neuro fuzzy inference system (ANFIS) for the first time. The conventional method of DATC is tedious and time consuming. DATC is concerned with calculating the maximum increase in point to point transfer such that the transient response remains stable and viable. The ATC information is to be continuously updated in real time and made available to market participants through an internet based Open Access Same time Information System (OASIS). The independent system operator (ISO) evaluates the transaction in real time on the basis of DATC information. The dynamic contingency screening method [1] has been utilized and critical contingencies are selected for the computation of DATC using the energy function based potential energy boundary surface (PEBS) method. The PEBS based DATC has been utilized to generate patterns for the intelligent techniques. The three different intelligent methods are tested on New England 68-bus 16 machine and 39-bus 10 machine systems and results are compared with the conventional PEBS method.
\end{abstract}

Keywords: DATC, dynamic contingency screening, intelligent techniques and power system deregulation

\section{Introduction}

The available transfer capability (ATC) of a transmission network refers to its unutilized transfer capability available for further transactions, over and above already committed usage, without violating system security constraints [2]. The determination of ATC should give power market participants enough information quickly to participate in the bidding process. The ATC depends on the variations of generation and demand in competitive power markets. Market participants need ATC information before bidding, in particular during system congestion. Fast ATC computation will help market participants make more efficient rounds of bidding. The ATC information is also very important for an ISO to determine the validity of the bidding results in power markets. The constraints required to determine ATC include static constraints such as line thermal limits, voltage limits and maximum loadability limits and dynamic constraints such as Transient and Small signal stability limits. ATC determined using the static constraints has been termed as static ATC, and that determined using both static and dynamic constraints, is termed as dynamic ATC (DATC). In many power systems, however, point to point transfer is not restricted by steady state limits, but by undesirable dynamic behavior following large disturbances. The post disturbance operating point certainly must be viable; but it is also important to ensure that the system can safely make the transition from the pre to the post distur-

$\dagger$ Corresponding Author: Department of Electrical Engineering, National Institute of Technology, Warangal - 506004 Andhra Pradesh India. (ch.venkaiah@gmail.com)

* Department of Electrical Engineering, National Institute of Technology, Warangal - 506004 Andhra Pradesh, India.

** ABB Limited, Maneja Vadodara, India

Received: October 24, 2009; Accepted: March 26, 2010 bance operating point. The computation of DATC presented by I. A. Hiskens et al. [3] is based on maximum increase in point-to-point power transfer so that the transient response remains stable.

Several Researchers have addressed the issue of ATC computation considering only static limits, such as line thermal limit, bus voltage limit and steady state stability limit. Various approaches have been proposed in the literature to compute static ATC such as continuation power flow, AC power flow, DC power flow, optimal power flow and sensitivity based methods. Very few methods have been reported in literature to determine DATC considering stability constraints such as small signal stability, transient stability limit and voltage stability limit. The fast evaluation of DATC considering stability limits is still a challenging task and this paper addresses one.

The dynamic voltage stability evolution mechanism of a power system is illustrated by Y. Cheng et al. [4], and quasi steady state approximation is applied in the determination of ATC with consideration of dynamic voltage stability constraints. An iterative approach, utilizing trajectory sensitivity concept, has been proposed by I. A. Hiskens et al. [5] for computing DATC. A static optimization based approach, including dynamic security constraints for the assessment of ATC, has been proposed by Enrico De Tuglie et al. [6]. Jonathan W Stahlhut et al. [7] attempted the method of deterministic evaluation of ATC using the stochasticalgebraic analytic technique based on two uncertainties viz. bus loading and transmission element status. Deqiang Gan et al. [8] presented a methodology that eliminates the need for repeated simulations to determine a transiently secure operating point and a mechanism of conversion of dynamic equations to numerically equivalent algebraic equations for integrating into standard OPF formulation. Yue Yuan et al. [9] proposed a novel method for integrating transient sta- 
bility constraints into ATC problem as an OPF based optimization problem. An interior point non-linear programming methodology is developed for evaluating DATC. Yan Xia et al. [10] developed an ATC calculation method which considers transient stability constraints and was solved using a direct non-linear primal-dual interior point method. Mohamed Shaaban et al. [11] investigated the evaluation of ATC under steady state security conditions using Benders decomposition and formulated an optimization problem with all normal operating conditions and contingency conditions of the system. The existence of stable equilibrium in post - disturbance system is only a necessary condition of system stability [3]. Thus, it is also important to ensure that the system can safely make the transition from the pre to post disturbance operation point. A model, using equilibrium equations as steady state constraints and a dot product concept given by Enrico De Tuglie et al. [6] as a rotor angle stability constraint, has been proposed by Xuemin Zhang et al. [12] to determine DATC. The sub optimal value of DATC has been computed by T. Jain et al. [13] using a structure preserving energy function model for different bilateral and multilateral transactions. The accurate ATC value has been obtained through few time domain simulations (TDS). In order to reduce the number of runs of TDSs and to obtain the transaction amount with a tolerable and positive energy margin, the Golden Bisection based interpolation method has been utilized. The evaluation of DATC by an optimization based method considering Hopf bifurcation limit in an electricity market having different bilateral as well as multilateral transactions has been proposed by T. Jain et al. [14]. The assessment of DATC by R. Gnanadass et al. [15] for practical power systems has been computed by using the fitness distance ratio particle swarm optimization (FDRPSO) algorithm, to determine the optimal dispatch of power producers with dynamic security constraints.

In this paper, the PEBS method based on an energy function algorithm has been considered for direct stability studies to calculate DATC. The proposed DATC method first performs numerical simulations to compute the energy margin using the PEBS method. By increasing the power at source bus and load at sink bus, the energy margin at each power step has been calculated. This process continues until the energy margin is less than the pre-specified threshold value. For the effective use of power systems under the deregulated environment, it is important to make a fast and accurate evaluation of the maximum ATC from a supply point to a demand point. A repetition of (N-1) contingencies with constraints of thermal limit, voltage stability and transient stability under multiple supply / demand scenarios are required for ATC assessment. Hence, the necessary computational effort of ATC calculation becomes very large. ATC assessment with transient stability constraints has a dominant part in the overall computational cost of an ATC assessment. Thus, transient stability assessment by time domain simulation method is a time consuming process. The dynamic contingency screening method is one of the counter measures for this computational problem. A novel dynamic contingency screening method is discussed by
Chingjun Fu et al. [1], Yoshinori Yamada et al. [16] and critical contingencies are selected. DATC between a given source sink pair is calculated for each critical contingency and the effective DATC is a minimum of all ATCs corresponding to critical contingencies. T. Jain et. al. [17] presented an approach based on a radial basis function neural network to rank the contingencies expected to cause steady state bus voltage violation. D. M. Vinod Kumar et. al. [18] computed ATC for real time applications for the first time using two different neural networks viz. i) BPA and ii) RBF by testing on New England 10 machine 39-bus system and compared with PEBS method. Azhar B. Khairuddin et. al. [19] proposed a novel method of ATC computation in a large power system from only three input variables through fuzzy modeling.

This paper attempts DATC computation using three different artificial intelligence (AI) techniques as an extension to the work presented in [18] for a larger power system and utilized the ANFIS intelligent technique for the first time in DATC computation. The contribution of the paper is the computation of DATC using three intelligent techniques for the first time to reduce the computation time for real time applications. In this paper BPA, RBF and ANFIS intelligent techniques have been utilized for computing DATC and tested on New England 16 machine 68-bus system [20] and New England 10 machine 39-bus system [21]. The results are compared with conventional methods of energy function based PEBS method.

The paper is organized as follows. The introduction of various methods of computation of DATC and their limitations for real time application is discussed in section 1 . The energy function based PEBS method for computation of critical clearing time, critical energy and energy margin is explained in detail in section 2. Section 3 deals with the algorithm for computation of PEBS based dynamic ATC. Section 4 introduces the proposed method of computing DATC using artificial intelligence techniques for the first time to reduce the computation time and for real time applications. Section 5 gives the simulated results of the three intelligent techniques viz. BPA, RBF and ANFIS applied to New England 16 machine and 10 machine systems and compared with the PEBS method.

\section{Energy Function based PEBS Method for Transient Stability Studies}

In transient stability, the computation of the critical clearing time $\left(t_{c r}\right)$ and energy margin (EM) is needed when the system is subjected to large disturbances. The energy function methods presented by M. A. Pai [22], Peter W Sauer et al. [23], Hsiao-Dong Chiang et al. [24] have proven to be reliable and are considered as promising tools in dynamic security assessment. The computation of maximum value of the potential energy $\left(\mathrm{V}_{\mathrm{cr}}\right)$ component of $\mathrm{V}(\mathrm{x})$ along the trajectory of $\dot{x}=f^{F}(x(t)) 0<t \leq t_{c l}$ (fault clearing time) with an initial condition $\mathrm{x}(\mathrm{o})=\mathrm{x}_{\mathrm{o}}$ is known as the potential energy boundary surface (PEBS) method. In this paper, the PEBS method has been considered to 
compute $V_{c r}, t_{c r}$ and EM.

In transient energy function technique, we must consider the model in two time frames, as follows:

\section{Faulted System}

a) $\dot{x}=f^{F}(x(t)) \quad 0<t \leq t_{c l}$

\section{Post Fault System}

a) $\dot{x}=f(x(t)) \quad t>t_{c l}$

In reality, the model is a set of differential - algebraic equations (DAE), i.e.,

$$
\begin{gathered}
\dot{x}=f^{F}(x(t), y(t)) \\
0=g^{F}(x(t), y(t)) \quad 0<t \leq t_{c l}
\end{gathered}
$$

The function ' $g$ ' represents the nonlinear algebraic equations of the stator and the network, while the differential equations represent the dynamics of the generating unit and its controls. There are methods like reduced order models, such as flux decay model and classical models have been presented. In the classical model representation, we can use either the preserve the network structure (structure preserving model) or eliminate the load buses (assuming constant impedance load) to obtain the internal node model. Structure preserving models involve nonlinear algebraic equations in addition to dynamic equations, and can incorporate non linear models leading to the concept of structure preserving energy function (SPEF) V(x,y), while models consisting of differential equations lead only to closed form types of energy functions $\mathrm{V}(\mathrm{x})$.

Analytical Lyapunov functions can be constructed only if the transfer conductances $\left(D_{i j}=0\right)$ are zero. Since these terms have to be accounted properly, the first integrals of motion of the system are constructed, and these are called energy functions. There are two options: to use either the relative rotor angle formulation or the Center of Inertia (COI) formulation. The latter option has been utilized since there are some advantages [22]. The resulting energy function is called the transient energy function (TEF) as the angles are referred to a COI. In this formulation, the angle of the COI is used as the reference angle, since it represents the "mean of motion" of the system. Although the resulting energy function is identical to $V(\delta, \omega)$ (using relative rotor angles), it has the advantage of being more symmetric and easier to handle in terms of the path dependent terms. Synchronous stability of all machines is judged by examining the angles referenced only to the COI instead of relative rotor angles. Modern literature invariably uses the COI formulation. The energy function in the COI notation, including $\mathrm{D}_{\mathrm{ij}}$ terms (transfer conductance) was proposed.

\subsection{Algorithm for Computation of Critical Clearing Time, Critical Energy and Energy Margin using PEBS method:}

Step 1. Compute stable equilibrium point $x_{s}$ from post fault system equation $\dot{x}=f(x(t)) \quad t>t_{c l}$

Step 2. Formulate $V(x)$ for post fault system. Generally, $V(x)$ is the sum of kinetic and potential energies of the post fault system, i.e., $V(x)=V_{K E}+V_{P E}$

Step 3. Computation of Vcr: In PEBS method $V_{\text {cr }}$ is obtained by integrating the faulted trajectory until the potential energy part $V_{P E}$ of $V(x)$ reaches a maximum $V_{P E}^{\max }$. This value is taken as $V_{c r}$ (critical energy) in the PEBS method.

Step 4. Calculating the time of instant $t_{c r}$ (critical clearing time) when $V(x)=V_{c r}$ on the faulted trajectory. In PEBS method, the faulted trajectory is already available while computing $V_{c r}$. Hence, the computation time is least for the PEBS method.

Step 5. Compute $V(x)=V_{c l}$ at Fault Clearing Time $t_{c l}$

Step 6. Compute Energy Margin (EM) as difference be tween $V_{c r}$ and $V_{c l}$ i.e. $\mathrm{EM}=V_{c r}-V_{c l}$

\section{Computation of PEBS based Dynamic ATC (DATC)}

\subsection{Dynamic Contingency Screening}

Fast contingency screening is expected to be an integral part of any practical on line dynamic security analysis (DSA). Ranking of contingencies requires the use of severity indices, and for DSA such an index must be a measure of stability. In static security analysis, it is well known that some indices work better than others for particular power systems and those combinations of indices usually work better than a single index. For DSA, which are still in its infancy, severity indices are more complex and very few have been tested for screening. Several indices are proposed for contingency screening in on line DSA [1]. These indices are based on the concepts of coherency, transient energy conversion between kinetic energy and potential energy, and three dot products of system variables. DATC has been found for each screened critical contingency, and the effective DATC is the minimum of all DATCs computed.

\subsection{Algorithm for Dynamic Contingency Screening using EM based PI method}

Step 1. Select the possible (N-1) contingencies; say there are $\mathrm{N}$ possible contingencies.

Step 2. For contingency $=1$, find the energy margin (EM) as discussed in section 2 and set its value as $\Delta V$

Step 3. While integrating the faulted system equations, find the difference between $V_{K E}$ and $V_{P E}$ at each time step, and the maximum value of these differences are to be noted. This maximum value is $\Delta V_{K E}$.

Step 4. Then compute $\Delta V_{n}=\Delta V / \Delta V_{K E} \quad \&$ Proposed Index $(\mathrm{PI})=1 / \Delta V_{n}$ where $\Delta V=$ Transient Energy Margin (The difference of the energy at the controlling unstable equilibrium point and at fault 
clearing); $\Delta V_{K E}=$ Kinetic Energy

Step 5. Find the PI for every contingency, and arrange them in descending order. If the PI is high, severity of contingency is high and it should be given first rank. Rank all N contingencies using the PI. Five critical contingencies are selected out of the $\mathrm{N}$ contingencies. These five critical contingencies affect the DATC between a pair of source and sink.

Dynamic contingency screening is an important step in the fast assessment of DATC. Accuracy of DATC computation depends on the accurate selection of the severe contingencies in the contingency list. The ranking of contingencies were based on the proposed index method and such an index must be a measure of stability. The critical contingencies are selected based on the cut off point between stable and unstable contingencies. If the proposed index is high, severity of contingency is high and it should be given first rank.

Contingency screening obtained by EM based PI method for the New England 16 machine 68-Bus System has been utilized for simulation. Here, $35(\mathrm{~N}-1)$ credible contingencies are considered and the five most severe critical contingencies are selected based on the performance index (PI) shown in Table 1.
Table 1. Selected Critical Contingencies

\begin{tabular}{c|c|c}
\hline \multicolumn{3}{c}{ New England 16 Machine 68-Bus System } \\
\hline Fault Bus & Line to be removed & Proposed Index \\
\hline 25 & 25 to 02 & 1.0669 \\
\hline 32 & 32 to 30 & 0.9351 \\
\hline 52 & 52 to 49 & 0.9197 \\
\hline 37 & 37 to 32 & 0.8775 \\
\hline 22 & 22 to 21 & 0.8276 \\
\hline
\end{tabular}

\subsection{Algorithm for Computation of DATC using EM based PEBS Method}

Step 1. Select a source bus and sink bus between which dynamic ATC is to be calculated.

Step 2. Select the first critical contingency, and read corresponding fault bus and line to be removed to clear fault.

Step 3. For the first contingency and the base case loadings, find the Energy Margin (EM).

While (EM $>$ Threshold value (0.1)),

Increase the source bus power and sink bus power with power step of 0.1 p.u. and compute EM.

Step 4. If (EM $<$ Threshold value), then DATC is the difference between source bus power after series of increments and base case source bus power.

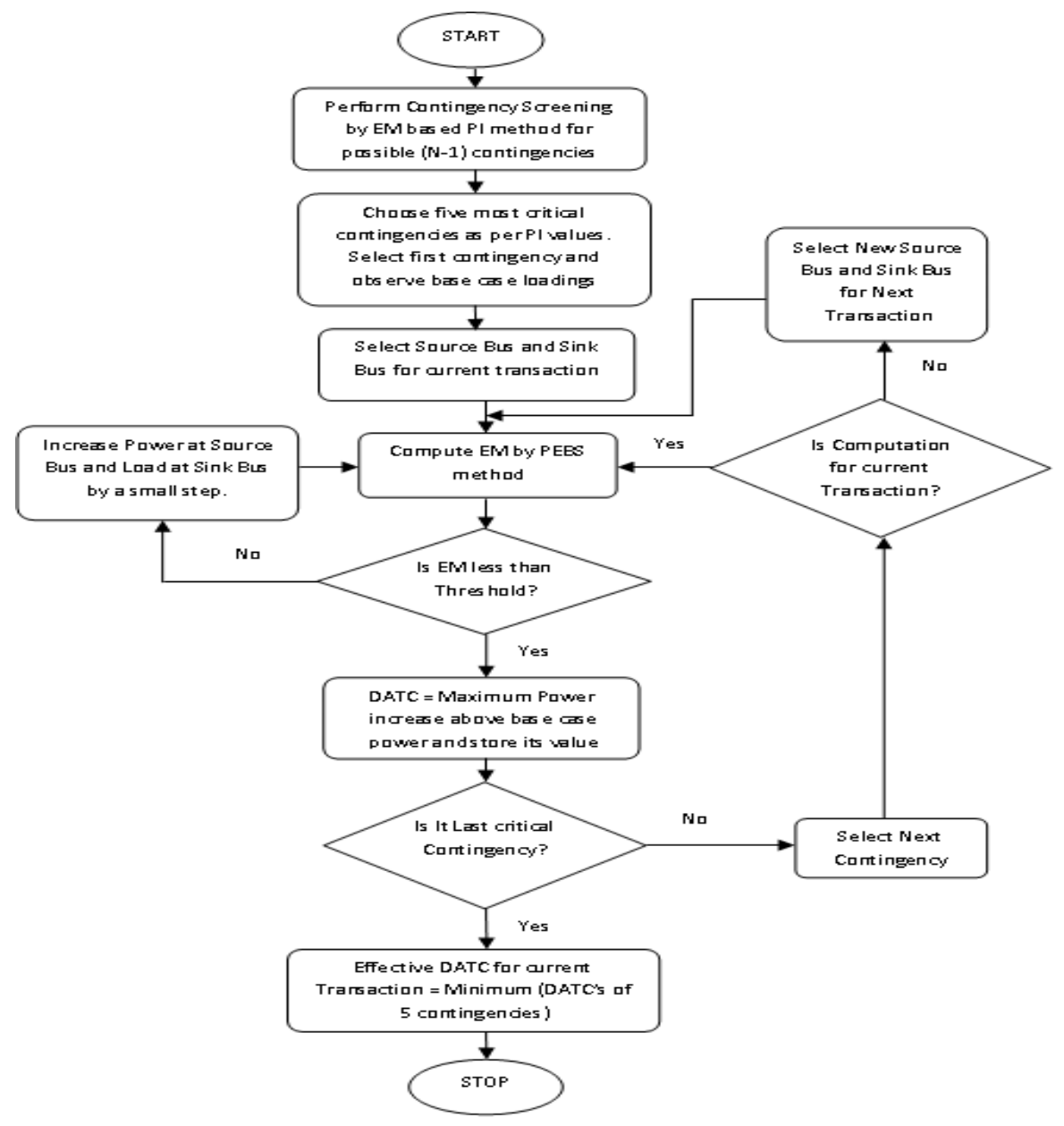

Fig. 1. Flow chart for PEBS based DATC computation. 
Step 5. Repeat the steps (3) and (4) and calculate DATC for each critical contingency.

Step 6. Effective DATC between given source sink pair is minimum of all DATCs, which corresponds to critical contingencies

The computation of PEBS based DATC is represented pictorially in the flow chart shown in Fig. 1.

After identifying the severe contingencies, the critical clearing time, critical energy and energy margin were computed using the PEBS method as discussed in section 2 and the values are shown in Table 2 at fault clearing time $\mathrm{T}_{\mathrm{cl}}=$ 0.15 seconds. Table 2 shows that the system remains in a secure state as the fault is cleared before the critical clearing time for all contingencies and sufficient energy margin is available to transact power during the contingency period. The critical clearing time, critical energy and the EM are varying with respect to fault position.

Table 2. $t_{c r}, V_{c r}$ and EM for selected critical faults on New England 16 machine 68-bus system, $\mathrm{Tcl}=0.15$ secs

\begin{tabular}{c|c|c|c|c}
\hline $\begin{array}{c}\text { Fault } \\
\text { Bus }\end{array}$ & $\begin{array}{c}\text { Line to be } \\
\text { removed }\end{array}$ & $\begin{array}{c}\text { Critical Clear- } \\
\left.\text { ing Time ( } \mathrm{T}_{\text {cr }}\right)\end{array}$ & $\begin{array}{c}\text { Critical } \\
\text { Energy } \\
\left.\text { ( } \mathrm{V}_{\text {cr }}\right)\end{array}$ & $\begin{array}{c}\text { Energy } \\
\text { Margin } \\
\text { (EM) }\end{array}$ \\
\hline 25 & 25 to 02 & 0.55 & 1.3589 & 1.2432 \\
\hline 32 & 32 to 30 & 0.57 & 1.2700 & 1.2689 \\
\hline 52 & 52 to 49 & 0.50 & 3.1200 & 3.0893 \\
\hline 37 & 37 to 43 & 0.42 & 1.1046 & 1.0996 \\
\hline 22 & 22 to 21 & 0.48 & 1.2744 & 1.2312 \\
\hline
\end{tabular}

The DATCs for different source and sink pairs of three transactions (T1, T2 and T3) each pertaining to five selected critical contingencies have been calculated and the results are presented in Table 3 . The five critical contingencies have affected the DATC between a chosen pair of source and sink based on the fault position.

Table 3. DATC(p.u) for different Transactions in the New England 16 machine 68-bus system

\begin{tabular}{c|c|c|c|c}
\hline \multirow{2}{*}{ Fault Bus } & \multirow{2}{*}{ Line to be removed } & \multicolumn{3}{|c}{ Dynamic ATC } \\
\cline { 3 - 5 } & & T1 & T2 & T3 \\
\hline 25 & 25 to 02 & 8.2 & 7.9 & 4.7 \\
\hline 32 & 32 to 30 & 6.7 & 9.0 & 8.1 \\
\hline 52 & 52 to 49 & 8.0 & 8.5 & 8.6 \\
\hline 37 & 37 to 32 & $\mathbf{3 . 1}$ & $\mathbf{3 . 3}$ & $\mathbf{3 . 2}$ \\
\hline 22 & 22 to 21 & 5.3 & 6.3 & 4.8 \\
\hline
\end{tabular}

T1: DATC is 3.1 p.u for Transaction from source bus (62) to sink bus (40) T2: DATC is 3.3 p.u for Transaction from source bus (58) to sink bus (28)

T3: DATC is 3.2 p.u for Transaction from source bus (60) to sink bus (18)

\section{Dynamic ATC Computation using Artificial Intelligent Techniques}

In the restructured power market, consumers can choose their power supplier; hence the transmission system becomes a critical element to transfer power. To utilize transmission systems effectively, the information about the ATC must be made known to ISOs. Hence, the information about the ATC should be made available on OASIS, which is accessible to consumers and utilities. ATC values posted on OASIS should be updated at a specified time interval. The value of ATC determines the further commercial activities of the system, and over cautious conservative estimation of it may lead to inefficient utilization of transmission networks. So, it must be calculated fast and accurately. ATC assessment with transient stability constraints (transient stability ATC) has a dominant part in overall computational costs of an ATC assessment, as transient stability assessment by time domain simulation method is a time consuming process. Even the calculation of DATC using the energy function based method will take more computation time. The original contribution of this paper is to reduce the computation time of DATC for real time applications using BPA, RBF and ANFIS intelligent techniques. The computation of DATC using intelligent techniques is represented pictorially in the flow chart shown in Fig. 2.

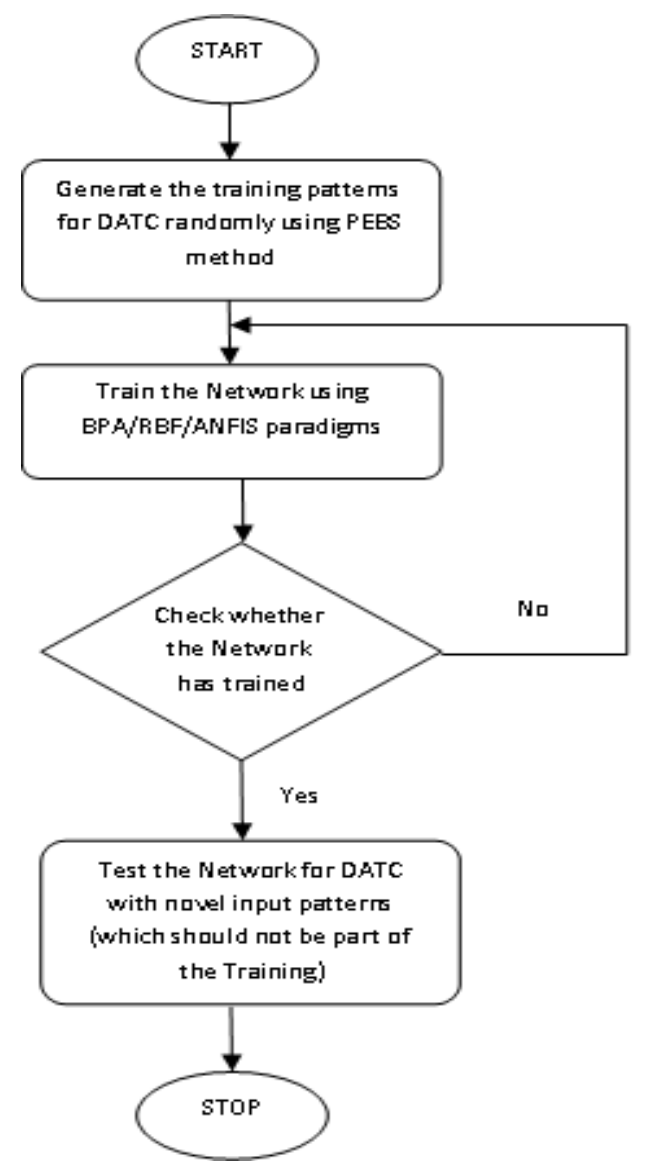

Fig. 2. A general flow chart of DATC computation using Intelligent Techniques.

\section{Simulations and Results}

In the restructured power markets, consumers can choose their power supplier. The transmission system be- 
comes a critical element of the power system. The information about the ATC should be made available on OASIS, which is accessible to consumers and utilities. ATC values posted on OASIS should be updated at specified time intervals. The value of ATC determines the further commercial activities of the system and over cautious conservative estimation of it may lead to inefficient utilization of transmission networks. So, it must be calculated fast and accurately. ATC assessment with transient stability constraints has a dominant part in the overall computational costs of an ATC assessment, as transient stability assessment by time domain simulation method is a time consuming process. Even calculation of DATC using the energy function based method will also take more time. To reduce the computational time, three artificial intelligent techniques viz. BPA, RBF and ANFIS have been utilized to compute the DATC.

A. Selection of Input variables: DATC between a given pair of source and sink buses in a large system is determined using five inputs. These are source bus injection $\left(P_{s}\right)$, loading index $(\gamma)$ for the base case, Fault Bus (FB), Base case Active Power Flow (P) in the line which is to be removed to clear the fault, and fault clearing time $\left(\mathrm{t}_{\mathrm{cl}}\right)$ are used for the BPA and RBF Neural Networks which is shown in Fig. 3. The BPA Neural Network utilized has one hidden layer with 11 neurons and it has been considered by hit and trial, which has provided minimum error. In the case of the RBF neural network, the number of hidden neurons selected as 205 with Gausian density function and used the same five inputs in the input layer as in BPA. The loading index $(\gamma)$ gives the operating condition of power systems by taking into account the load demands at all the buses. The loading index is defined as $\gamma=\left(\sum \mathrm{P}_{\mathrm{di}}\right) / \mathrm{A}_{\max }$ for all $\mathrm{i}=1$ to $\mathrm{N}$ buses; where $\mathrm{P}_{\mathrm{di}}$ is demand (MW) at bus $\mathrm{i}$ and $\mathrm{A}_{\max }$ is the thermal loadability (MVA) of the line having the highest limit in the system.

In the case of ANFIS, all five inputs are fuzzified and the number of attributes chosen for each input is three linguistic variables viz. low, medium and high, shown in Fig. 4.

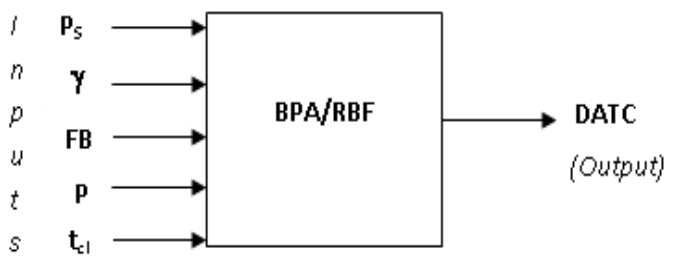

Fig. 3. Neural Network Inputs and Output.

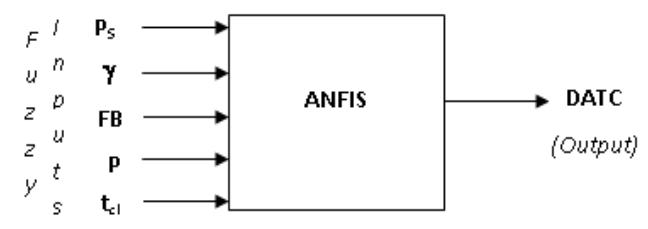

Fig. 4. ANFIS Inputs and Output.
B. Implementation: The New England 16 machine 68-bus system [20] and the New England 10 machine 39-bus system [21] data has been used to compare the performance of the proposed method with that of the energy function based PEBS method for DATC determination. The original Bus Numbers of both systems have been modified for grouping of the generator buses together for an effective solution. The pair of buses 36 (source) and 21 (sink) for 10 machine 39-bus system and pair of buses 54 (source) and 48 (sink) for 16 machine 68-bus systems are considered for illustrating the computation of DATC.

C. Generation of Patterns: The Training and testing patterns are generated using the energy function based PEBS method by treating bus 36 as source and bus 21 as sink for 10 machine 39-bus system and min-max random variables limits of the five inputs are: $\mathrm{P}_{\mathrm{s}}(0$ to 0.2$) ; \gamma(0.23$ to $0.36) ; \mathrm{FB}(12$ to 39$) ; \mathrm{P}(-2.6$ to 4.3$) ; \mathrm{t}_{\mathrm{cl}}(0.1$ to 0.4$)$. The training and testing patterns are generated by treating bus 54 as source and bus 48 as sink for 16 machine 68bus system and min max limits of the five inputs are: $\mathrm{P}_{\mathrm{s}}(0$ to 0.2$) ; \gamma(0.52$ to 0.65$) ; \mathrm{FB}(17$ to 68$) ; \mathrm{P}(-2.4$ to $1.97) ; \mathrm{t}_{\mathrm{cl}}(0.1$ to 0.4$)$. These patterns are generated by randomly varying the five inputs with in the min and max limits.

D. Training: Training sets provided to the neural network are representative of the whole state space of concern so that the trained system has the ability of generalization. The training patterns for the New England 10 machine 39-bus system and 16 machine 68-bus systems are generated considering three-phase faults at different buses, fault clearing times and loadings. The 550 patterns are generated out of these 500 patterns and were used to train the network.

E. Testing: The trained neural network was tested using 50 new patterns composed of different fault cases, fault clearing times and loadings. None of these patterns were used in the training of the neural network.

\subsection{Case A: New England 10 Machine 39-Bus System}

The DATC values, between source bus (36) and sink bus (21), computed by using intelligent techniques are given in Table 4 and it shows the comparison of three methods viz. BPA, RBF and ANFIS with the PEBS method [23]. It is found that the ANFIS algorithm is best compared to the other two methods as the ANFIS algorithm computed DATC values tracked the actual values with minimum error.

The normalized errors were estimated as |(PEBS DATC) - (BPA/RBF/ANFIS DATC)|/(PEBS DATC). The normalized error for BPA, RBF and ANFIS has been shown in Table 4 respectively for New England 10 machine 39-bus and their maximum error values were found to be 0.33884 , 0.16069 and 0.13440 for BPA, RBF and ANFIS respectively in comparison with PEBS DATC values. The ANFIS method of computing DATC was found to have minimum error. The number of rules and parameters related to fuzzy 
Table 4. DATC(p.u) between Source Bus (36) and Sink Bus (21) for the New England 10 Machine 39-bus system

\begin{tabular}{|c|c|c|c|c|c|c|c|}
\hline $\begin{array}{c}\text { Test } \\
\text { Pattern }\end{array}$ & $\begin{array}{c}\text { PEBS } \\
\text { DATC[23] }\end{array}$ & BPA DATC & $\begin{array}{c}\text { BPA } \\
\text { Normalized } \\
\text { Error }\end{array}$ & RBF DATC & $\begin{array}{c}\text { RBF } \\
\text { Normalized } \\
\text { Error }\end{array}$ & $\begin{array}{l}\text { ANFIS } \\
\text { DATC }\end{array}$ & $\begin{array}{c}\text { ANFIS } \\
\text { Normalized } \\
\text { Error }\end{array}$ \\
\hline 1 & 7.30000 & 7.10040 & 0.02734 & 7.25770 & 0.00579 & 7.30000 & 0.00000 \\
\hline 2 & 6.10000 & 6.34610 & 0.04034 & 6.09260 & 0.00121 & 6.07810 & 0.00359 \\
\hline 3 & 2.90000 & 2.89740 & 0.00090 & 3.12320 & 0.07697 & 2.87580 & 0.00834 \\
\hline 4 & 7.50000 & 7.50610 & 0.00081 & 7.47350 & 0.00353 & 7.42600 & 0.00987 \\
\hline 5 & 7.30000 & 7.25900 & 0.00562 & 7.37950 & 0.01089 & 6.31890 & 0.13440 \\
\hline 6 & 3.10000 & 4.03080 & 0.30026 & 3.04920 & 0.01639 & 3.07410 & 0.00835 \\
\hline 7 & 6.30000 & 7.10060 & 0.12708 & 6.10560 & 0.03086 & 6.29980 & 0.00003 \\
\hline 8 & 4.00000 & 4.14590 & 0.03648 & 4.05710 & 0.01428 & 3.97590 & 0.00602 \\
\hline 9 & 6.30000 & 7.04190 & 0.11776 & 6.30480 & 0.00076 & 6.27990 & 0.00319 \\
\hline 10 & 5.60000 & 5.81270 & 0.03798 & 5.61550 & 0.00277 & 5.55300 & 0.00839 \\
\hline 11 & 5.50000 & 5.84820 & 0.06331 & 5.67690 & 0.03216 & 5.48150 & 0.00336 \\
\hline 12 & 7.09990 & 7.05280 & 0.00663 & 7.80950 & 0.09995 & 7.04470 & 0.00777 \\
\hline 13 & 4.10000 & 4.10160 & 0.00039 & 4.08500 & 0.00366 & 4.07160 & 0.00693 \\
\hline 14 & 4.20000 & 4.19700 & 0.00071 & 4.17960 & 0.00486 & 4.18770 & 0.00293 \\
\hline 15 & 6.50000 & 7.10040 & 0.09237 & 6.26000 & 0.03692 & 6.45830 & 0.00642 \\
\hline 16 & 7.80000 & 8.05460 & 0.03264 & 7.60410 & 0.02512 & 7.74970 & 0.00645 \\
\hline 17 & 6.00000 & 5.98480 & 0.00253 & 6.24290 & 0.04048 & 5.95830 & 0.00695 \\
\hline 18 & 6.70000 & 6.60540 & 0.01412 & 6.21840 & 0.07188 & 6.68220 & 0.00266 \\
\hline 19 & 3.80000 & 2.51240 & 0.33884 & 3.73090 & 0.01818 & 3.77440 & 0.00674 \\
\hline 20 & 5.80000 & 6.27060 & 0.08114 & 5.80450 & 0.00078 & 5.78530 & 0.00253 \\
\hline 21 & 6.70000 & 7.10040 & 0.05976 & 6.76210 & 0.00927 & 6.66080 & 0.00585 \\
\hline 22 & 6.50000 & 6.28540 & 0.03302 & 6.93340 & 0.06668 & 6.45700 & 0.00662 \\
\hline 23 & 5.70000 & 5.75000 & 0.00877 & 6.28970 & 0.10346 & 5.65910 & 0.00718 \\
\hline 24 & 7.40000 & 7.39880 & 0.00016 & 7.60690 & 0.02796 & 7.24140 & 0.02143 \\
\hline 25 & 6.20000 & 6.27620 & 0.01229 & 6.24770 & 0.00769 & 6.14490 & 0.00889 \\
\hline 26 & 3.20000 & 3.27060 & 0.02206 & 3.44080 & 0.07525 & 3.19640 & 0.00113 \\
\hline 27 & 5.70000 & 6.27080 & 0.10014 & 5.69450 & 0.00096 & 5.67840 & 0.00379 \\
\hline 28 & 7.00000 & 7.12910 & 0.01844 & 6.92640 & 0.01051 & 6.94390 & 0.00801 \\
\hline 29 & 6.40000 & 6.43150 & 0.00492 & 6.40300 & 0.00047 & 6.37590 & 0.00377 \\
\hline 30 & 3.00000 & 2.99900 & 0.00033 & 2.93060 & 0.02313 & 2.97200 & 0.00933 \\
\hline 31 & 6.00000 & 6.00010 & 0.00002 & 5.99850 & 0.00025 & 5.95730 & 0.00712 \\
\hline 32 & 6.30000 & 6.21190 & 0.01398 & 6.42470 & 0.01979 & 6.28950 & 0.00167 \\
\hline 33 & 6.00000 & 6.25220 & 0.04203 & 5.93450 & 0.01092 & 5.99010 & 0.00165 \\
\hline 34 & 6.30000 & 6.69100 & 0.06206 & 6.46810 & 0.02668 & 6.29310 & 0.00110 \\
\hline 35 & 0.60005 & 0.61732 & 0.02878 & 0.58200 & 0.03008 & 0.55961 & 0.06739 \\
\hline 36 & 2.40000 & 2.51000 & 0.04583 & 2.34200 & 0.02417 & 2.38640 & 0.00567 \\
\hline 37 & 6.10000 & 5.84820 & 0.04128 & 6.26720 & 0.02741 & 6.06880 & 0.00511 \\
\hline 38 & 7.90000 & 7.24270 & 0.08320 & 7.97650 & 0.00968 & 7.84210 & 0.00733 \\
\hline 39 & 6.70000 & 6.70030 & 0.00004 & 6.74000 & 0.00597 & 6.65820 & 0.00624 \\
\hline 40 & 6.70000 & 6.92950 & 0.03425 & 5.98960 & 0.10603 & 6.54290 & 0.02345 \\
\hline 41 & 0.70004 & 0.69938 & 0.00094 & 0.72105 & 0.03001 & 0.69887 & 0.00167 \\
\hline 42 & 7.20010 & 7.39480 & 0.02704 & 6.10160 & 0.15257 & 7.18030 & 0.00275 \\
\hline 43 & 2.90000 & 2.51000 & 0.13448 & 2.89880 & 0.00041 & 2.88720 & 0.00441 \\
\hline 44 & 6.80000 & 6.39890 & 0.05899 & 6.63870 & 0.02372 & 6.77630 & 0.00349 \\
\hline 45 & 6.60000 & 5.84470 & 0.11444 & 5.64210 & 0.14514 & 6.54760 & 0.00794 \\
\hline 46 & 7.09990 & 7.10060 & 0.00010 & 6.53330 & 0.07980 & 7.07280 & 0.00382 \\
\hline 47 & 6.60000 & 7.15030 & 0.08338 & 6.59030 & 0.00147 & 6.55550 & 0.00674 \\
\hline 48 & 7.50000 & 6.84960 & 0.08672 & 7.88410 & 0.05121 & 7.42590 & 0.00988 \\
\hline 49 & 6.20000 & 6.56720 & 0.05923 & 6.13830 & 0.00995 & 6.20000 & 0.00000 \\
\hline 50 & 1.60000 & 1.59090 & 0.00569 & 1.85710 & 0.16069 & 1.58470 & 0.00956 \\
\hline
\end{tabular}

model are independent of the system size for Adaptive Neuro Fuzzy Inference System (ANFIS). The computation times for computing DATC for 50 testing patterns were found to be 0.021 seconds, 0.022 seconds and 0.016 seconds for BPA, RBF and ANFIS respectively. Hence, the Adaptive Neuro Fuzzy Inference System (ANFIS) method computes DATC very fast and accurately and can be applied to larger systems for real-time applications. The comparisons of three intelligent techniques with the PEBS method is shown in Fig. 5. Among the three methods, the ANFIS method is best compared to the other two methods as it is tracking the actual values with minimum error in the 10 machine system. 


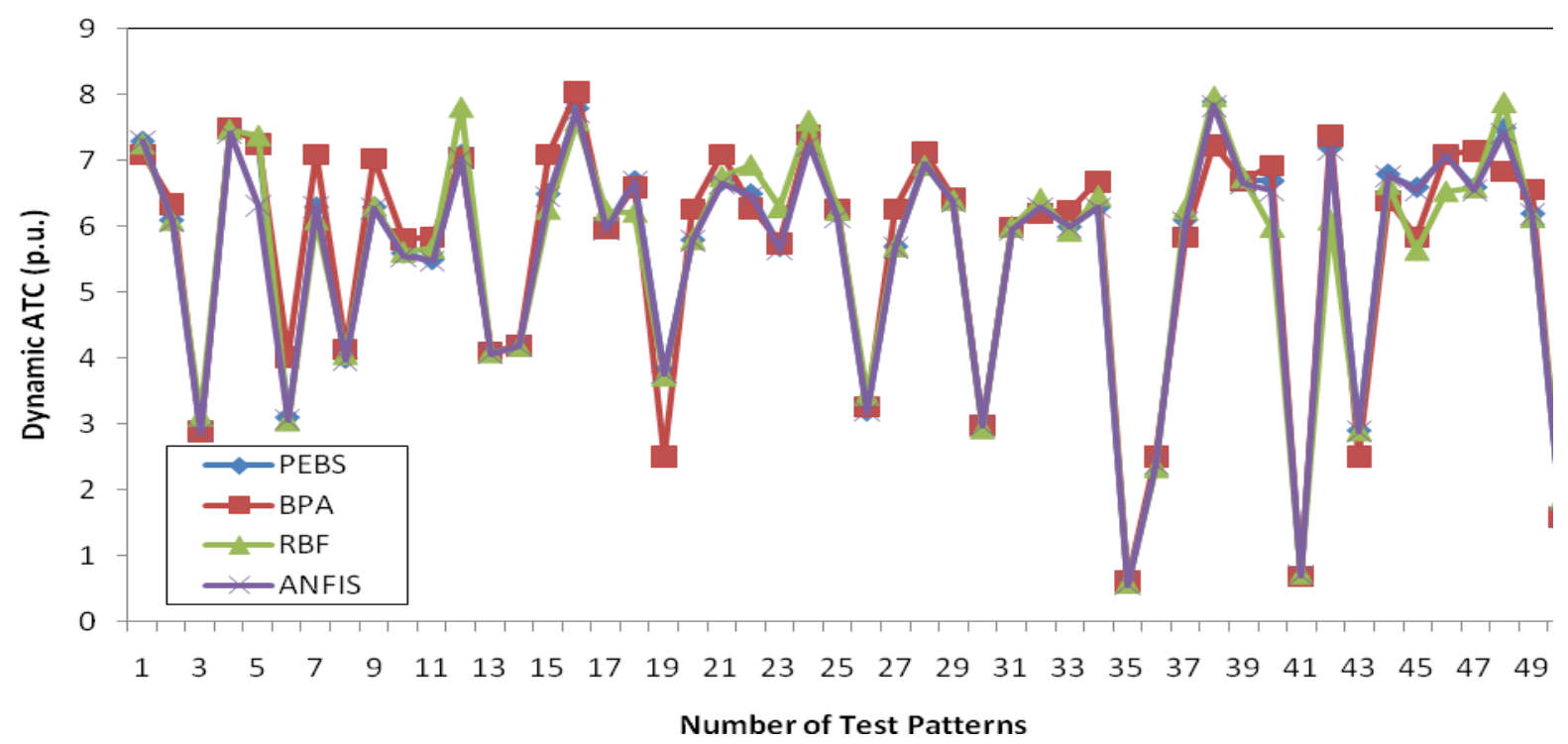

Fig. 5. DATC comparison between PEBS and BPA, RBF \& ANFIS for 10 machine 39 bus system.

\subsection{Case B: New England 16 Machine 68-Bus System}

The DATC values, between source bus (54) and sink bus (48), computed by using intelligent techniques are given in Table 5 and it shows the comparison of three methods viz. BPA, RBF and ANFIS with the PEBS method [23]. It is found that the ANFIS algorithm is best compared to the other two methods as the ANFIS algorithm computed DATC values are tracking the actual values with minimum error. The normalized errors were estimated as | (PEBS DATC) - (BPA/RBF/ANFIS DATC)|/ (PEBS DATC). The normalized error for BPA, RBF and ANFIS has been shown in Table 5 respectively for New England 16 machine 68-bus system and their maximum error values were found to be $0.0700,0.0355$ and 0.0001 for BPA, RBF and ANFIS respectively in comparison with PEBS DATC val- ues. The ANFIS method of computing DATC was found to have minimum error. The number of rules and parameters related to fuzzy model are independent of the system size for the adaptive neuro fuzzy inference system (ANFIS). The computation times for computing DATC for 50 testing patterns were found to be 0.022 seconds, 0.022 seconds and 0.017 seconds for BPA, RBF and ANFIS respectively. Hence, the ANFIS method computes DATC very fast and accurately and can be applied to larger systems for realtime applications. The comparisons of three intelligent techniques with the PEBS method is shown in Fig. 6. Among the three methods, the ANFIS method is best compared to the other two methods as it is tracking the actual values with minimum error in the 16 machine test system. The testing time of the intelligent techniques viz. BPA, RBF and ANFIS have been compared in terms of CPU time (in seconds) for computing DATC for 50 testing pat-

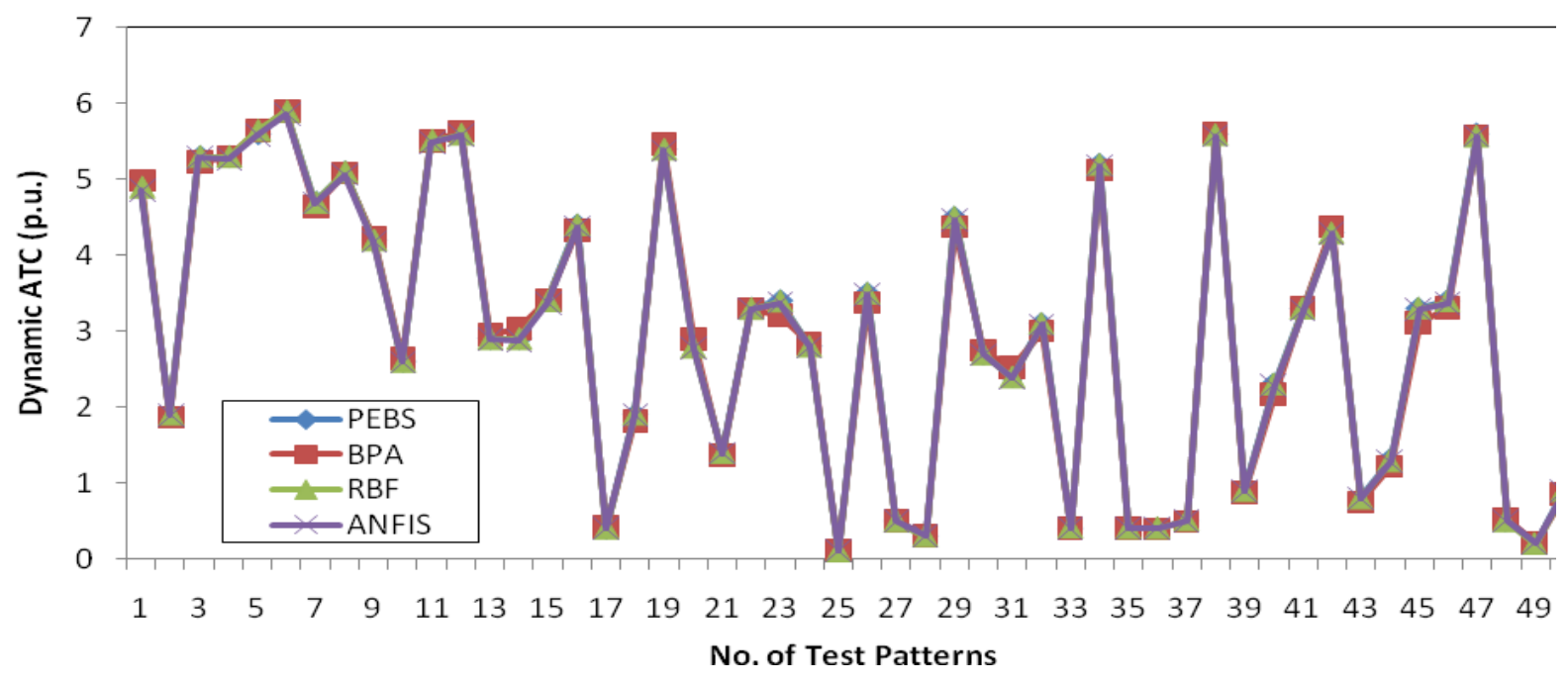

Fig. 6. DATC comparison between PEBS and BPA, RBF \& ANFIS for 16 machine 68 bus system. 
Table 5. DATC(p.u) between Source Bus (54) and Sink Bus (48) for the New England 16 Machine 68-bus system

\begin{tabular}{|c|c|c|c|c|c|c|c|}
\hline $\begin{array}{c}\text { Test } \\
\text { Pattern }\end{array}$ & $\begin{array}{c}\text { PEBS DATC } \\
{[23]}\end{array}$ & BPA DATC & $\begin{array}{l}\text { BPA Normal- } \\
\text { ized Error }\end{array}$ & RBF DATC & $\begin{array}{l}\text { RBF Normal- } \\
\text { ized Error }\end{array}$ & $\begin{array}{l}\text { ANFIS } \\
\text { DATC }\end{array}$ & $\begin{array}{c}\text { ANFIS Normal- } \\
\text { ized Error }\end{array}$ \\
\hline 1 & 4.9 & 4.9809 & 0.0165 & 4.8971 & 0.0006 & 4.8600 & 0.0082 \\
\hline 2 & 1.9 & 1.8618 & 0.0201 & 1.9009 & 0.0005 & 1.8871 & 0.0068 \\
\hline 3 & 5.3 & 5.2197 & 0.0152 & 5.3023 & 0.0004 & 5.2862 & 0.0026 \\
\hline 4 & 5.3 & 5.2946 & 0.0010 & 5.3029 & 0.0005 & 5.2694 & 0.0058 \\
\hline 5 & 5.6 & 5.6348 & 0.0062 & 5.6492 & 0.0088 & 5.5834 & 0.0030 \\
\hline 6 & 5.9 & 5.8942 & 0.0010 & 5.9011 & 0.0002 & 5.8650 & 0.0059 \\
\hline 7 & 4.7 & 4.6320 & 0.0145 & 4.6986 & 0.0003 & 4.6928 & 0.0015 \\
\hline 8 & 5.1 & 5.0775 & 0.0044 & 5.1002 & 0.0000 & 5.0679 & 0.0063 \\
\hline 9 & 4.2 & 4.2256 & 0.0061 & 4.1992 & 0.0002 & 4.1801 & 0.0047 \\
\hline 10 & 2.6 & 2.6322 & 0.0124 & 2.5984 & 0.0006 & 2.5930 & 0.0027 \\
\hline 11 & 5.5 & 5.5034 & 0.0006 & 5.5183 & 0.0033 & 5.4913 & 0.0016 \\
\hline 12 & 5.6 & 5.6270 & 0.0048 & 5.5970 & 0.0005 & 5.5906 & 0.0017 \\
\hline 13 & 2.9 & 2.9459 & 0.0158 & 2.8998 & 0.0001 & 2.8890 & 0.0038 \\
\hline 14 & 2.9 & 3.0238 & 0.0427 & 2.9010 & 0.0003 & 2.8725 & 0.0095 \\
\hline 15 & 3.4 & 3.3992 & 0.0002 & 3.4009 & 0.0003 & 3.3666 & 0.0098 \\
\hline 16 & 4.4 & 4.3224 & 0.0176 & 4.4002 & 0.0000 & 4.3736 & 0.0060 \\
\hline 17 & 0.4 & 0.4218 & 0.0545 & 0.3993 & 0.0018 & 0.3960 & 0.0100 \\
\hline 18 & 1.9 & 1.8062 & 0.0494 & 1.9022 & 0.0012 & 1.8863 & 0.0072 \\
\hline 19 & 5.4 & 5.4594 & 0.0110 & 5.3990 & 0.0002 & 5.3829 & 0.0032 \\
\hline 20 & 2.8 & 2.8898 & 0.0321 & 2.7880 & 0.0043 & 2.7768 & 0.0083 \\
\hline 21 & 1.4 & 1.3557 & 0.0316 & 1.3976 & 0.0017 & 1.3868 & 0.0094 \\
\hline 22 & 3.3 & 3.2897 & 0.0031 & 3.2971 & 0.0009 & 3.2929 & 0.0022 \\
\hline 23 & 3.4 & 3.2095 & 0.0560 & 3.3963 & 0.0011 & 3.3756 & 0.0072 \\
\hline 24 & 2.8 & 2.8295 & 0.0105 & 2.7994 & 0.0002 & 2.7897 & 0.0037 \\
\hline 25 & 0.1 & 0.1022 & 0.0220 & 0.0981 & 0.0190 & 0.0993 & 0.0070 \\
\hline 26 & 3.5 & 3.3724 & 0.0365 & 3.4998 & 0.0001 & 3.4819 & 0.0052 \\
\hline 27 & 0.5 & 0.5005 & 0.0010 & 0.4982 & 0.0036 & 0.4953 & 0.0094 \\
\hline 28 & 0.3 & 0.2974 & 0.0087 & 0.3006 & 0.0020 & 0.2990 & 0.0033 \\
\hline 29 & 4.5 & 4.3696 & 0.0290 & 4.5012 & 0.0003 & 4.4688 & 0.0069 \\
\hline 30 & 2.7 & 2.7418 & 0.0155 & 2.6987 & 0.0005 & 2.6892 & 0.0040 \\
\hline 31 & 2.4 & 2.5156 & 0.0482 & 2.3942 & 0.0024 & 2.3873 & 0.0053 \\
\hline 32 & 3.1 & 2.9981 & 0.0329 & 3.1004 & 0.0001 & 3.0838 & 0.0052 \\
\hline 33 & 0.4 & 0.4032 & 0.0080 & 0.4142 & 0.0355 & 0.3971 & 0.0073 \\
\hline 34 & 5.2 & 5.1139 & 0.0166 & 5.1974 & 0.0005 & 5.1836 & 0.0032 \\
\hline 35 & 0.4 & 0.4030 & 0.0075 & 0.4072 & 0.0180 & 0.3970 & 0.0075 \\
\hline 36 & 0.4 & 0.3915 & 0.0213 & 0.4065 & 0.0162 & 0.3965 & 0.0088 \\
\hline 37 & 0.5 & 0.4897 & 0.0206 & 0.5076 & 0.0152 & 0.4977 & 0.0046 \\
\hline 38 & 5.6 & 5.6029 & 0.0005 & 5.5927 & 0.0013 & 5.5730 & 0.0048 \\
\hline 39 & 0.9 & 0.8652 & 0.0387 & 0.8966 & 0.0038 & 0.8914 & 0.0096 \\
\hline 40 & 2.3 & 2.1686 & 0.0571 & 2.3001 & 0.0000 & 2.2795 & 0.0089 \\
\hline 41 & 3.3 & 3.2966 & 0.0010 & 3.3006 & 0.0002 & 3.2760 & 0.0073 \\
\hline 42 & 4.3 & 4.3703 & 0.0163 & 4.2953 & 0.0011 & 4.2839 & 0.0037 \\
\hline 43 & 0.8 & 0.7440 & 0.0700 & 0.7990 & 0.0013 & 0.7932 & 0.0085 \\
\hline 44 & 1.3 & 1.2140 & 0.0662 & 1.2875 & 0.0096 & 1.2873 & 0.0098 \\
\hline 45 & 3.3 & 3.1062 & 0.0587 & 3.3010 & 0.0003 & 3.2878 & 0.0037 \\
\hline 46 & 3.4 & 3.3043 & 0.0281 & 3.3995 & 0.0001 & 3.3661 & 0.0100 \\
\hline 47 & 5.6 & 5.5662 & 0.0060 & 5.5722 & 0.0050 & 5.5722 & 0.0050 \\
\hline 48 & 0.5 & 0.5126 & 0.0252 & 0.4996 & 0.0008 & 0.4995 & 0.0010 \\
\hline 49 & 0.2 & 0.2023 & 0.0115 & 0.2006 & 0.0030 & 0.1993 & 0.0035 \\
\hline 50 & 0.9 & 0.8557 & 0.0492 & 0.8982 & 0.0020 & 0.8935 & 0.0072 \\
\hline
\end{tabular}

terns, is found from Table 6 that all the proposed intelligent techniques took very less time to compute the DATC. The simulation was carried out on Pentium ${ }^{\circledR} 4 \mathrm{CPU}, 3.00 \mathrm{GHz}$ $496 \mathrm{MB}$ of RAM personal computer.
Table 6. Comparison of CPU Time (in seconds)

\begin{tabular}{c|c|c|c}
\hline Test System & BPA & RBF & ANFIS \\
\hline 39-Bus System & 0.021 & 0.022 & 0.016 \\
\hline 68-Bus System & 0.022 & 0.022 & 0.017 \\
\hline
\end{tabular}




\section{Conclusion}

In this paper, DATC has been computed for real-time applications using intelligent techniques viz., back propagation algorithm (BPA), neural network, radial basis function (RBF), neural network and adaptive neuro fuzzy inference system (ANFIS) and results are compared with the potential energy boundary surface (PEBS) method for the first time. The proposed method has been tested on New England 10 machine 39-bus system and New England 16 machine 68-bus system. The ANFIS method of computing dynamic ATC was found to have minimum error and minimum CPU time for both the systems. The training of RBF neural network requires less computation time as compared to the BPA, since only the second layer weights have to be calculated using an error signal. The number of rules and parameters related to the fuzzy model are independent of the system size for the adaptive neuro fuzzy inference system (ANFIS). Hence, the ANFIS method computes DATC very fast and accurately and can be applied to larger systems for real-time applications.

\section{References}

[1] Chingjun Fu and Anjan Bose, "Contingency Ranking Based on Severity Indices in Dynamic Security Analysis," IEEE Transactions on Power System, Vol. 14, No. 3, pp. 980-986, Aug. 1999.

[2] North American Electric Reliability Council (NERC), Available Transfer Capability Definitions and Determination, NERC Report, June 1996.

[3] Ian A. Hiskens, M. A. Pai and P. W. Sauer, "Dynamic ATC," IEEE Power Engineering Society Winter meeting, Singapore, Vol. 3, pp. 1629, January 23-27, 2000.

[4] Y. Cheng, T. S. Chung, C. Y. Chung and C. W. Yu, "Incorporation of Dynamic Voltage Stability Constraints in the ATC Determination of Power System," Sixth International Conference on Advances in Power System Control, Operation and Management, APSCOM, 2003, Hong Kong, pp. 619-623, November, 2003.

[5] I. A. Hiskens, M. A. Pai and P. W. Sauer, "An Iterative Approach to Calculating Dynamic ATC", Bulk Power System Dynamics and Control IV-Restructuring, Santorino, Greece, pp. 585-590, Aug. 24-28, 1998.

[6] Enrico De Tuglie, Maria Dicorato, Massimo La Scala and Pierangelo Scarapellini. "A Static Optimization Approach to Assess Dynamic Available Transfer Capability," IEEE Transactions on Power Systems, Vol. 15, No. 3, pp. 1069-1076, August 2000.

[7] Jonathan W. Stahlhut and Gerald Thomas Heydt, "Stochastic-Algebraic Calculation of Available Transfer Capability," IEEE Transactions on Power Systems, Vol.22, No.2, pp. 616-623, May 2007.

[8] Deqiang Gan, Robert J. Thomas, Ray D. Zimmerman, "Stability Constrained Optimal Power Flow," IEEE Transactions on Power System, Vol. 15, No. 2, pp. 535- 540, May 2000.

[9] Yue Yuan, Junji Kubokawa, Takeshi Nagata and Hi- roshi Sasaki, “A solution of Dynamic Available Transfer Capability by means of Stability Constrained Optimal Power Flow," 2003 IEEE Bologna Power Tech conference, Bologna, Italy, June 23-26, 2003.

[10] Yan Xia, Ka Wing Chan, Mingbo Liu and Jie Wu, "Calculation of Available Transfer Capability with Transient Stability Constraints," 2004 IEEE International Conference on Electric Utility Deregulation, Restructuring and Power Technologies (DRPT2004), Hong Kong, pp. 128-132, April 2004.

[11] Mohamed Shaaban, Yixin Ni and Felix F. Wu, "Available Transfer Capability Evaluation by Decomposition," IEEE Power Engineering Society Summer meeting, Vol. 2, pp. 1122-1126, 2001.

[12] Xuemin Zhang, Y. H. Song, Qiang Lu and Shengwei Mei, "Dynamic available transfer capability (ATC) Evaluation by Dynamic Constrained Optimization," IEEE Transactions on Power System, Vol. 19, No. 2, pp. 1240-1242, May 2004.

[13] T. Jain, S. N. Singh and S. C. Srivastava, "Dynamic Available Transfer Capability Computation using a hybrid approach," IET Generation, Transmission \& Distribution, Vol. 2, No. 6, pp. 775-788, 2008.

[14] T. Jain, S. N. Singh and S. C. Srivastava, "Dynamic Available Transfer Capability Evaluation Considering Hopf Bifurcation Limit," Fifteenth National Power System Conference (NPSC-2008, IIT Bombay, pp. 55- 60), December 2008.

[15] R. Gnanadass and Venkataramana Ajjarapu, "Assessment of Dynamic Available Transfer Capability using FDR PSO Algorithm," ELEKTRIKA, Vol. 10, No. 1, pp. 20-25, 2008.

[16] Yoshinori Yamada, Masaki Nagata and Kazuyuki Tanaka, "An Energy Function based Contingency Screening Method for ATC Assessment with Transient Stability Constraints," IEEE, pp. 886-890, 2002.

[17] T. Jain, L. Srivastava and S. N. Singh, "Fast Voltage Contingency Screening Using Radial Basis Function Neural Network," IEEE Transactions on Power Systems, Vol. 18, No. 4, pp.1359-1366, November 2003.

[18] D. M. Vinod Kumar and Ch. Venkaiah, "Dynamic Available Transfer Capability (DATC) Computation using Intelligent Techniques," IEEE Power India Conference 2008 POWERCON 2008, October 12-15, 2008.

[19] Azhar B. Khairuddin, S. Shahnawaz Ahmed, M. Wazir Mustafa, Abdullah A. Mohd. Zin and Hussein Ahmad, "A Novel Method for ATC Computations in a Large-Scale Power System," IEEE Transactions on Power Systems, Vol. 19, No. 2, pp. 1150-1158, May 2004.

[20] http://read.pudn.com/downloads75/sourcecode/ app/278205/DATA16M.M .htm, 2008.

[21] http://psdyn.ece.wisc.edu/IEEE_benchmarks/, 2008.

[22] M. A. Pai, "Energy Function Analysis for Power System Stability," Kluwer academic publishers, Boston/ London, 1989.

[23] Peter W. Sauer and M. A. Pai, "Power systems dy- 
namics and stability," Pearson Education 2003.

[24] Hsiao-Dong Chiang, Chia-Chi-Chu and Gerry Cauley, "Direct Stability Analysis of Electric Power Systems using Energy Functions: Theory, Applications and Perspective," IEEE Proceedings, Vol. 83, No. 11, pp. 1497-1529, November 1995.

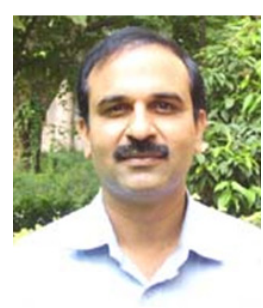

Ch. Venkaiah received his B.Tech degree in electrical and electronics engineering from JNTUCOE Kakinada, his M.Tech degree in computer science and engineering from National Institute of Technology (NIT), Warangal and he is currently pursuing his Ph.D. degree in Electrical Engineering at NIT, Warangal. He is an Associate Professor of Electrical Engineering at NIT, Warangal. His current research interests include power system deregulation and artificial intelligence technique applications in power systems.

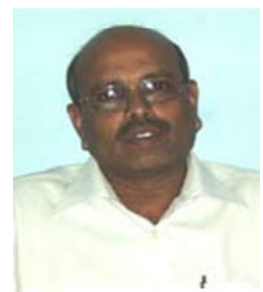

D. M. Vinod Kumar received his B.E. degree in electrical engineering, his M.E. degree in Power Systems Engineering from University College of Engineering, Osmania University, Hyderabad, Andhra Pradesh, India, and his Ph.D. degree in electrical engineering from the Indian Institute of Technology (IIT), Kanpur, India. At present, he is Dean (Academic Affairs) and Professor of Electrical Engineering at the National Institute of Technology, Warangal. His current research interests include power system deregulation, artificial intelligence technique applications in power systems, power system stability, energy management systems (EMS) and flexible AC transmission systems (FACTS).
K. Murali received his B.Tech degree in electrical engineering from Kakatiya Institute of Technology and Science (KITS), Warangal, and his M.Tech degree in Power Systems Engineering from the National Institute of Technology, Warangal Andhra Pradesh, India. At present, he is working as a domain engineer with ABB Limited, Maneja, Vadodara, India. His current research interests include power system protection and power system stability. 